\title{
Fertility control: the role of magnesium transporters in pollen development
}

\author{
Bernd Mueller-Roeber ${ }^{1}$, Samuel Arvidsson ${ }^{1}$ \\ ${ }^{1}$ University of Potsdam, Department of Molecular Biology, Karl-Liebknecht-Straße 24-25, Haus 20, D-14476 Potsdam-Golm, Ger- \\ many
}

Cell Research (2009) 19:800-801. doi: 10.1038/cr.2009.82; published online 2 July 2009

Magnesium $\left(\mathrm{Mg}^{2+}\right)$ is essential for many plant processes. It is particularly important for photosynthesis, where it is the central element of the chlorophyll molecules. Magnesium is also a constituent of many enzymes and serves as an activator of others, including DNA polymerases, protein kinases, and phosphatases. Intracellular $\mathrm{Mg}^{2+}$ concentration is precisely controlled by $\mathrm{Mg}^{2+}$ uptake, translocation across intracellular membranes and efflux [1]. Surprisingly, however, the molecular mechanisms underlying these processes in higher plants are still largely unknown. Recently, two reports $[2,3]$ disclosed functions of two $\mathrm{Mg}^{2+}$ transporters in pollen development. Pollen grains are the male gametes in flowering plants.

$\mathrm{Mg}^{2+}$ transporters of the CorA family appear to be the primary transport systems mediating both influx and efflux of $\mathrm{Mg}^{2+}$. CorA-type transporters are widely distributed in bacteria, fungi, animals and in plants (see below). The crystal structure of the CorA transporter from the bacterium Thermotoga maritima has recently been elucidated revealing a unique structure with a large, acidic $\mathrm{N}$-terminal domain and two transmembrane domains in the $\mathrm{C}$-terminal region [4]. The currently best studied eukaryotic homologues are those from bud-

Correspondence: Bernd Mueller-Roeber E-mail: bmr@uni-potsdam.de ding yeast (Saccharomyces cerevisiae), where the MRS2 and LPE10 proteins are targeted towards the inner mitochondrial membrane, while ALR1, ALR2 and MNR2 transporters are located in the plasma membrane [5].

Higher plant transporters of the CorA/MRS2 family were first identified in 2000, when Schock et al. [6] reported a gene family of ten members in Arabidopsis thaliana, named AtMRS2-1 to AtMRS2-10. These authors also demonstrated that AtMRS2-1 functionally complements Mrs2-deficient yeast restoring intramitochondrial $\mathrm{Mg}^{2+}$ concentration to the wild-type level.

Functional evidence for $\mathrm{Mg}^{2+}$ transport was also provided by Li et al. [7] who in 2001 discovered the same gene family, but named it as AtMGT (for Arabidopsis thaliana magnesium transporter). Most members of the AtMGT gene family were shown by RT-PCR to be expressed throughout the plant, with the exception of AtMGT5 which was only expressed in flowers, and AtMGT8 that was not expressed in stem tissue. AtMGT1 functionally complemented a bacterial (Salmonella typhimurium) mutant called MM281 that lacks three $\mathrm{Mg}^{2+}$ transport systems (CorA, MgtA and MgtB). AtMGT1 turned out to be a high-affinity transporter for $\mathrm{Mg}^{2+}$. Among divalent cations it was highly selective for $\mathrm{Mg}^{2+}$. AtMGT10 complemented a yeast mutant, lacking both ALR1 and ALR2 genes [7], although it localizes to the chloroplast envelope membrane when expressed in plant cells, indicating a role in $\mathrm{Mg}^{2+}$ uptake and translocation into chloroplasts [8]. Recently the first low-affinity $\mathrm{Mg}^{2+}$ transporter, AtMGT7a, was identified in higher plants. Two different transcripts are produced from the AtMGT7 gene; AtMGT7a transcript is expressed in all tissues analyzed, whereas the slightly shorter AtMGT7b transcript is present in roots and flowers [9]. Only AtMGT7a complemented the Salmonella MM281 mutant, whereas AtMGT7b, which encodes a protein lacking a 15 -amino acid region, did not.

The biological roles of all above mentioned $\mathrm{Mg}^{2+}$ transporters are still unknown although ectopic expression of AtMGT1 in Nicotiana benthamiana conferred improved tolerance to aluminum [10]. However, a physiological function was recently discovered for AtMGT5 which represents a dual-functional $\mathrm{Mg}^{2+}$ transporter that mediates uptake and efflux of $\mathrm{Mg}^{2+}$ in a concentrationdependent manner when expressed in the MM281 strain [2]. At low (i.e., micromolar) concentration of $\mathrm{Mg}^{2+}$ the AtMGT5 protein serves as an importer, whereas at high (millimolar) $\mathrm{Mg}^{2+} \mathrm{lev}-$ els it facilitates efflux of the divalent cation. Expression of AtMGT5-GFP fusion protein in transgenic Arabidopsis plants labeled mitochondria (verified by MitoTracker Red CMXRos staining), suggesting that it shuttles $\mathrm{Mg}^{2+}$ be- 
tween the mitochondrial compartment and the cytoplasm. Promoter-reporter ( $\beta$-glucuronidase) fusion studies revealed exclusive expression of AtMGT5 in anthers at earlier stages of flower development, in both gametophytic and sporophytic tissues [2]. Interestingly, when AtMGT5 was mutated by T-DNA insertion, pollen formation was strongly impaired in hemizygous mutants; homozygous atmgt 5 mutants were never obtained, indicating lethality of a complete knock-out of the AtMGT5 gene.

The story became even more fascinating when Chen et al. [3] described a second $\mathrm{Mg}^{2+}$ transporter, AtMGT9, which also affected pollen viability. AtMGT9 supported the growth of the bacterial MM281 mutant at $500 \mu \mathrm{M}$ $\mathrm{Mg}^{2+}$, but not at $10 \mu \mathrm{M} \mathrm{Mg}^{2+}$, reminiscent of a low-affinity transporter. Tracer inhibition studies, that utilize the fact that $\mathrm{Mg}^{2+}$ and $\mathrm{Ni}^{2+}$ can be transported by the same CorA-type transporters with similar kinetics, revealed that AtMGT9 is able to transport $\mathrm{Mg}^{2+}$ at physiological concentrations normally found in plant cells, whereas the transport of other divalent ions - trace elements - appeared unlikely due to their much lower levels prevalent in plants. Transcriptional activity of AtMGT9 in anthers followed a distinct temporal and spatial pattern. Its expression was evenly distributed throughout the anther early in development and was then concentrated in the tapetum, the tissue nursing pollens during their formation. AtMGT9 expression vanished when the tapetum was degraded and resumed again during pollen maturation. As with AtMGT5, disruption of AtMGT9 led to pollen abortion, and only hemizygous mutants survived. Crossing experiments proved that the mutated atmgt 9 allele could not be transmitted through the pollen due to lack of fertility [3].

The works by Luan and co-workers $[2,3]$ show a tight connection between $\mathrm{Mg}^{2+}$ transporter function and pollen development for the first time, although we do not know in detail how the two transporters sustain pollen formation and thus fertility. One model supposes that both proteins are located in mitochondria (though the localization of AtMGT9 still has to be investigated) but function independently to contribute to mitochondrial $\mathrm{Mg}^{2+}$ homeostasis. Alternatively, both proteins may constitute subunits of a hetero-oligomeric transporter. However, as both AtMGT5 and AtMGT9 seemingly form functional transporters when individually expressed in bacterial cells, it appears likely that functional (homo-oligomeric) transporters can also form in planta. Another scenario is that AtMGT9 resides in a non-mitochondrial membrane to exert its transport function jointly with mitochondrial AtMGT5. None of these models can be excluded at present.

A further interesting asset comes from the fact that both magnesium transporter genes follow distinct, partially overlapping tempo-spatial activity patterns during anther development. Identifying the transcription factors that govern their expression will contribute to our understanding of the regulatory networks that control pollen viability through the function of AtMGT5 and
AtMGT9 transporters.

\section{References}

1 Shaul O. Magnesium transport and function in plants: the tip of the iceberg. Biometals 2002; 15:309-323.

2 Li LG, Sokolov LN, Yang YH, et al. A mitonchondrial magnesium transporter functions in Arabidopsis pollen development. Mol Plant 2008; 1:675-685.

3 Chen J, Li LG, Liu ZH, et al. Magnesium transporter AtMGT9 is essential for pollen development in Arabidopsis. Cell Res 2009; 19:887-898.

4 Maguire ME. The structure of CorA: a $\mathrm{Mg}^{2+}$-selective channel. Curr Opin Struct Biol 2006; 16:432-438.

5 Gardner RC. Genes for magnesium transport. Curr Opin Plant Biol 2003; 6:263-267.

6 Schock I, Gregan J, Steinhauser S, et al. A member of a novel Arabidopsis thaliana gene family of candidate $\mathrm{Mg}^{2+}$ ion transporters complements a yeast mitochondrial group II intron-splicing mutant. Plant J 2001; 24:489-501.

7 Li L, Tutone AF, Drummond RS, et al. A novel family of magnesium transport genes in Arabidopsis. Plant Cell 2001; 13:2761-2775.

8 Drummond RSM, Tutone A, Li YC, Gardner RC. A putative magnesium transporter AtMRS2-11 is localized to the plant chloroplast envelope membrane system. Plant Sci 2006; 170:7889.

9 Mao DD, Tian LF, Li LG, et al. AtMGT7: an Arabidopsis gene encoding a lowaffinity magnesium transporter. $J$ Integr Plant Biol 2008; 50:1530-1538.

10 Deng W, Luo K, Li D, et al. Overexpression of an Arabidopsis magnesium transport gene, AtMGT1, in Nicotiana benthamiana confers Al tolerance. J Exp Bot 2006; 57:4235-4243. 